\title{
Perioperative management of a patient with carnitine palmitoyltransferase 2 deficiency
}

\author{
Máximo M. ${ }^{1}$, Pereira M. ${ }^{1}$
}

Hospital Prof Doutor Fernando Fonseca, Dept of Anaesthesiology, Amadora, Portugal

\section{Background}

Carnitine palmitoyltransferase 2 (CPT-2) deficiency is a rare condition that results from a defective mitochondrial fatty acid oxidation. It is the most commonly inherited metabolic cause of recurrent rhabdomyolysis in adults. Fasting and stress are the usual triggers and propofol, neuromuscular blocking drugs and volatile anesthetics have also been implicated. Accompanying cardiomyopathy or conduction defects can occur. Careful perioperative management of these patients is of great importance to prevent disease exacerbation and rhabdomyolysis-related acute kidney injury (AKI).

\section{Case Report}

- ASA II, 43 year-old male patient;

- Known CPT-2 deficiency and a past history of rhabdomyolysis with severe AKI, as well as mild episodes of muscle pain and weakness induced by exercise and stress;

- He was submitted to an elective stapled haemorrhoidopexy;

- Spinal anesthesia with bupivacaine $(5 \mathrm{mg})$ was performed with insufficientblock. General balanced anesthesia was then induced with fentanyl $(0,05 \mathrm{mg})$ and propofol $(180 \mathrm{mg})$ and maintenance was made with sevoflurane $2 \%$;

- Perioperative management included individualized analgesic and fluid therapy, minimizing fasting and monitoring glycemia and creatine kinase (CK) levels;

- The postoperative period occurred uneventfully. Glucose levels were $159 \mathrm{mg} / \mathrm{dL}$ and CK was moderately raised $(605 \mathrm{U} / \mathrm{L})$, as expected after surgery;

- He was discharged at the first postoperative day.

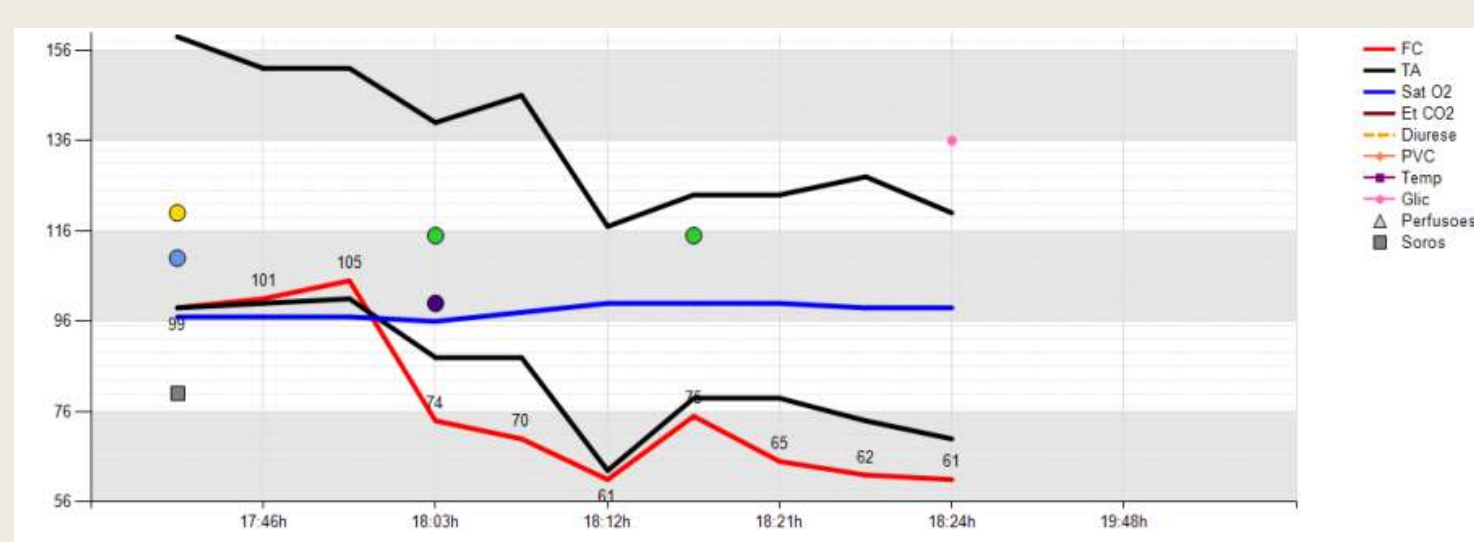

Figure 1: Intraoperative patient's monitoring

\section{Discussion}

The ideal anesthetic strategy in CPT-2 deficiency is still not defined and there are only around 300 reports worldwide ${ }^{1,2}$.

Minimizing fasting period, providing adequate carbohydrate intake and reducing surgical stress is always required as there is no etiological therapy. Past history of stressors may guide the approach. Additionally, patients' disease-related comorbidities and metabolic status must be carefully assessed.

Although local anesthesia is preferred ${ }^{1}$, general anesthesia can be safely performed. An action plan in case of massive rhabdomyolysis should be predefined.

\section{Learning Points}

Perioperative management of CPT-2 deficiency should be guided by patients' past history and identified stressors.

An optimized preventive strategy is essential and allows for safe general anesthesia in these patients. Perioperative massive rhabdomyolysis is a possible event and should be anticipated. 